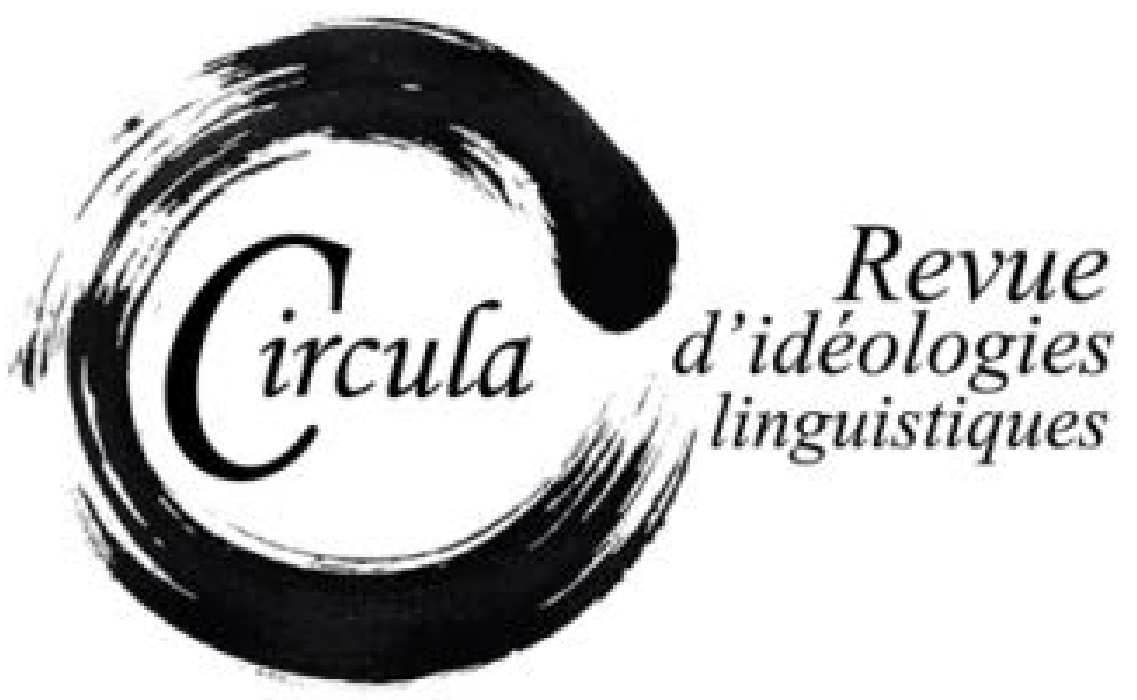

TITRE: LINGUISTICA “INGENUA” IN UNA RUBRICA LINGUISTICA DELLA STAMPA ITALIANA

Auteur(s): Giuliana Fiorentino, Università degli Studi del Molise, Campobasso

Revue: CiRcula, NumÉro 6

PAgES: $138-163$

ISSN: 2369-6761

Directeurs: Wim Remysen, SABIne SCHWARZE et JUAN ANTONIO EnNiS

URI: HTTP://HDL.HANDLE.NET/11143/11918

DOI: HTTPS://DOI.ORG/10.17118/11143/11918 


\section{Linguistica "ingenua" in una rubrica linguistica della stampa italiana}

Giuliana Fiorentino, Università degli Studi del Molise, Campobasso giuliana.fiorentino@unimol.it

Riassunto: La linguistica spiegata dai parlanti - chiamata linguistica ingenua, popolare, e in molti altri modi - costituisce un capitolo interessante, ancorché sfuggente, della rappresentazione sociale della lingua, cioè dell'ideologia linguistica di una comunità. In questo articolo si analizzano i discorsi dei lettori di una rubrica linguistica che appare su un quotidiano nazionale italiano online. La ricerca ricostruisce da un lato l'idea di norma linguistica e di errore di cui i lettori sono portatori attraverso le richieste di chiarimenti su fatti di lingua, e dall'altro la diffusione del metalinguaggio grammaticale tra i lettori della rubrica. La conclusione della ricerca mostra una visione abbastanza rigida e tradizionale sia della norma linguistica sia del linguaggio specialistico utilizzato per parlare di fatti di lingua.

Parole chiave: ideologia linguistica; linguistica ingenua; metalinguaggio; norma linguistica

Abstract: Linguistics explained by native speakers, also called naïve linguistics or folk linguistics, amateur linguistics, and in many other ways, is an interesting chapter, albeit elusive, of the social representation of language, i.e. language ideology of a community. In this article we analyze the discourses of the readers of a blog that appears on a national online Italian newspaper. The research discusses on one hand the idea of linguistic norm and of error which readers carry through their queries for clarification of language facts, and on the other hand the spread among non-linguists of the linguistic meta-language for talking about grammar. The conclusion of the research shows a fairly rigid view of the idea of linguistic norm and a very traditional meta-language used to talk about language facts.

Keywords: linguistic ideology; folk linguistics; metalanguage; standard language 


\section{Introduzione}

L'ideologia linguistica (Heath 1989; Woolard 1992, 1998; Blommaert 1999, 2006; Gal 2002; Leone 2011) è un tema di ricerca sviluppato nell'ambito degli studi linguistici, ma anche sociologici e antropologici, che attrae da anni studiosi di diverse scuole:

La recherche sur les idéologies linguistiques a connu un développement considérable au cours des dernières décennies. Depuis les années 1970 et 1980, le concept d'idéologie n'a de cesse d'être convoqué pour éclaircir les liens existant entre pratiques linguistiques (et discursives) et pratiques sociales (et culturelles) dans plusieurs travaux en sciences humaines et sociales. Il s'est ainsi progressivement imposé comme une notion-clé permettant de réunir des réflexions autant sur les idées qui circulent à propos du fonctionnement de la langue que sur la place que la langue occupe dans la société. (Remysen/Schwarze 2015: p. 1)

Una prospettiva particolare al suo interno è rappresentata dal quadro teorico dell'immaginario linguistico ${ }^{1}$. Per immaginario linguistico si intende la rappresentazione sociale della lingua all'interno di una comunità e il modo in cui essa interferisce con l'evoluzione della lingua e con l'evoluzione della stessa nozione di norma linguistica (Remysen 2011: 48). L'immaginario linguistico, nella definizione di Houdebine, si costituisce a partire dall'interscambio tra norme linguistiche oggettive e soggettive. Mentre le prime sono le norme di funzionamento oggettivamente ricostruibili in una lingua o norme di tipo statistico, quindi misurabili, le seconde sono l'insieme di valutazioni e giudizi che gli utenti esprimono e applicano; esse interagiscono con la loro visione della lingua e dell'evoluzione linguistica, con questioni comunicative e persino identitarie.

Uno dei luoghi in cui è possibile osservare tale rappresentazione sociale della lingua e considerare la visione più o meno scientifica o più o meno scolastica e tradizionale che dei fatti di lingua hanno i parlanti non specialisti è rappresentato dalle rubriche linguistiche che quotidiani o siti web specializzati hanno creato per instaurare un dialogo con i lettori-utenti. Tali rubriche consentono ai lettori di porre dei quesiti ai quali risponde una redazione di esperti di lingua e grammatica italiana. In particolare le rubriche linguistiche consentono di osservare l'immaginario linguistico di non specialisti appassionati di fatti di lingua.

In questo articolo si analizza l'ideologia linguistica di parlanti italiani non professionisti così come emerge dai quesiti e dai commenti che i lettori inviano a una di queste rubriche linguistiche, pubblicata sul quotidiano La Repubblica online. La ricerca si focalizza su alcuni punti che possono essere così sintetizzati: di che cosa si occupa prioritariamente la rubrica linguistica? Qual è la terminologia linguistica usata dagli utenti? Qual è la lista di problemi e dubbi linguistici più ricorrenti considerando le diverse aree della grammatica e i livelli dell'analisi linguistica? Quali sono i concetti ingenui (paro-

1. Si vedano Houdebine 1985 e 2002; per un'applicazione del quadro teorico alle cronache linguistiche sulla stampa si rimanda a Remysen 2011. 
la, gruppo di parole, frase) utilizzati dai lettori? E infine: quale ideologia della lingua si può ricostruire, in particolare rispetto a concetti come norma e deviazione dalla norma?

Se l'obiettivo primario dell'indagine è delineare l'ideologia linguistica o immaginario linguistico degli utenti, un obiettivo ulteriore consiste nel verificare la diffusione del linguaggio settoriale della linguistica (spesso innovativo rispetto alla tradizione grammaticale scolastica) o la persistenza della terminologia tradizionale nella cultura del parlante non professionista. Se ne può vedere un caso nell'estratto qui riportato come esempio (1):

(1) vorrei sapere come si comportano in analisi logica le locuzioni avverbiali. Grazie (Dubbi sull'italiano 6, del 4/12/2014)

dove risultano attestati i termini tecnici, benché scolastici, di analisi logica e locuzione avverbiale. Questi dati costituiscono materiali originali di indagine anche in vista di un rinnovamento della terminologia metalinguistica con finalità didattiche.

\section{Linguistica ingenua}

L'idea di contrapporre la visione della lingua dei non specialisti (ideologia linguistica dei parlanti comuni), a quella dei linguisti di professione (la linguistica scientifica), non è nuova in linguistica, prova ne sono i diversi termini con cui si designano le idee sulla lingua dei non professionisti: linguistica ingenua, naif, popolare, laica, profana, folk.

Prima di passare all'analisi del corpus di dati, si propongono brevemente, e senza velleità di esaustività, alcune di queste discussioni qui raccolte sotto il termine unificante di linguistica ingenua.

Si può partire dal manuale di Sapir Il Linguaggio (1921) in cui si discute la realtà psicologica dell'unità linguistica parola. Sapir osserva come anche nei parlanti nativi americani, che sono parlanti di lingue solo orali, si registra una nozione psicologica di 'parola', e che però quest'ultima risulta diversa dalla realtà psicologica che la parola riveste in parlanti competenti in una lingua anche scritta (1921 [1969: 33-34]). Questo argomento per Sapir serve a rafforzare l'idea che ci sia una «validità psicologica della parola» anche al di fuori delle costruzioni del linguista. Tale posizione fa individuare una «linguistica del parlante» non cólto, diversa dalla linguistica ufficiale. La stessa posizione si ritrova alcuni anni dopo nello stesso Sapir per giustificare l'esistenza di un'altra importante unità linguistica, cioè il fonema. In un famoso articolo del 1933 infatti si parla della realtà psicologica dei fonemi per tutti i parlanti, non soltanto per i linguisti (Sapir 1933).

2. D’ora in poi la fonte verrà omessa perché tutti gli esempi citati sono estratti dai post Dubbi sull'italiano 6 e Dubbi sull'italiano 7. 
Ad una conoscenza implicita e ingenua della lingua fa riferimento anche Giorgio Graffi, linguista italiano di scuola generativista, in un articolo del 1991 e poi nel suo manuale di Sintassi (1994) quando definisce le nozioni centrali della sintassi (parola, frase, gruppo di parole, parti del discorso, ecc.) basandosi sull'intuizione dei parlanti e sulla conoscenza implicita della lingua, cioè basandosi su «criteri osservativi molto neutri», e senza ricorrere inizialmente a una teoria generale del linguaggio (Graffi 1994: 42). Lo stesso Graffi definisce ingenui tali concetti «Parleremo quindi, riferendoci al contenuto intuitivo di nozioni come "frase", "parola", o "gruppo di parole", di concetti sintattici ingenui» (Graffi 1994: 26).

In un famoso articolo del 1992 Dixon elenca cinque casi in cui parlanti nativi incontrati durante il lavoro sul campo - quindi degli informanti - spiegano allo stesso Dixon questioni grammaticali relative alla propria lingua materna. In questo contesto lo studioso definisce questo tipo di esperienza linguistica naïve e parla di «spiegazioni di linguistica naïve» (Dixon 1992: p.?). Dixon dimostra come, in assenza di un opportuno metalinguaggio, il parlante nativo ricorra a spiegazioni «laterali» per arrivare comunque a comunicare correttamente il suo sentire linguistico al linguista di professione.

In un articolo recente Albano Leoni (2015) ricorre invece all'espressione ortografia ingenua per discutere le scelte ortografiche rilevate in un corpus napoletano di scritture esposte di varia tipologia (avvisi, graffiti, scritte commerciali, annunci funebri). Non interessa in questa sede ripercorrere l'analisi che di tali scritte fa lo studioso, quanto mostrare come il concetto di 'ingenuità' funzioni ancora per indicare l'idea che il parlante comune ha dei fatti di lingua e di come spesso tale visione ingenua possa rendere conto di aspetti non banali della lingua. L'ortografia che tali scritte manifestano, infatti, corrisponde all'«immagine fonica che i napoletano hanno del loro dialetto quando questo non sia filtrato attraverso l'ortografia canonica» (Albano Leoni 2015: 57).

Nel mondo francofono un termine corrispondente a quello di linguistica ingenua è linguistique populaire. A tale tema ad esempio sono dedicati due numeri monografici della rivista Pratiques, i numeri 139/140 del 2008. Dopo l'introduzione di Achard-Bayle et Paveau (2008) che spiegano il perché della scelta monografica, seguono articoli in cui si parla di aspetti della teorizzazione spontanea deIl'argomentazione (Doury 2008), di linguistica popolare applicata alla struttura della conversazione (Schmale 2008), di aspetti contrastivi della linguistica popolare in ambito francofono e germanofono (Stegu 2008), di aspetti teorici generali e definizioni di chi siano i 'non linguisti' (Paveau 2008) o di che cosa si occupi la linguistica popolare (Preston 2008), ed infine della realtà culturale dei nuovi stati dell'Europa dell'Est e della linguistica di nuovi pseudo-scienziati (Seriot et alii 2008).

3. Nel caso discusso da Albano Leoni le scelte ortografiche ingenue, non modellate sui canoni ortografici del napoletano letterario, nella loro eliminazione sistematica delle vocali atone (esempio l'ipocoristico 'siringaro' che diventa srngr) sarebbero compatibili con una classificazione del dialetto tra le lingue isoaccentuali e non tra quelle isosillabiche come l'italiano. Questo aspetto viene invece oscurato dai testi letterari che modellano l'ortografia del dialetto su quella dell'italiano per rendere visibile il legame etimologico (Albano Leoni 2015: 52-57). 
In ambito americano ricorre invece il termine di folk linguistics e il dibattito su questo argomento viene riaperto in modo vivace da Henry Hoenigswald nel 1964 durante una conferenza di sociolinguistica presso la University of California a Los Angeles (UCLA) (Hoenigswald 1966).

In ambito tedesco si parla di Laienlinguistik ('linguistica laica', Antos, 1996; Demel 2006, 2007, Jaeckel 2006, Osthus 2006).

\section{Il corpus}

I dati su cui si basano le considerazioni che seguono provengono dalla rubrica aperta dal linguista Massimo Arcangeli a partire dal giugno del 2009 su La Repubblica online (http://linguista.blogautore.repubblica.it/). Pur non essendo l'unica del genere, questa rubrica ha delle caratteristiche che l'hanno resa più interessante ai fini di questa ricerca4 ${ }^{4}$.

La struttura testuale della rubrica è quella di un blog in cui si distinguono un contenuto principale (post), pubblicato dalla redazione, che appare con il nickname Linguista, e i commenti degli utenti, che sono pubblicati con i diversi nickname scelti dagli utenti all'atto di registrarsi. Ai fini di questa ricerca la parte più interessante del blog è costituita dai numerosi commenti che seguono i post redazionali. All'interno dei commenti però interviene anche la redazione e fornisce risposte e ulteriori commenti, tessendo una sorta di dialogo con gli utenti. Tutti i post della redazione appaiono con il nickname Linguista, ma alla fine di ciascun testo compare il nome dell'autore di quello specifico testo (Arcangeli o uno dei collaboratori della redazione).

La rubrica, che non ha una cadenza regolare nel pubblicare i post, spazia su tutti i possibili ambiti della linguistica italiana discutendo di problemi che riguardano i vari livelli della lingua o questioni più ampie (norma e uso, aspetti teorici). Periodicamente la rubrica pubblica un post dal titolo Dubbi sull'italiano? Risponde il linguista (ne sono apparsi in tutto 7, l'ultimo risale al dicembre 2015') in cui si sollecitano gli utenti del blog a segnalare incertezze e curiosità relative alla norma linguistica italiana e nei quali, dunque, si riversano le domande dei lettori, senza un tema prefissato. Si è concentrata l'attenzione soprattutto sui post dedicati ai ‘dubbi linguistici', perché sono quelli che rivelano meglio

4. Altre rubriche analoghe che avrebbero potuto essere considerate e che possono costituire materia di confronto futuro sono la rubrica Consulenza linguistica, presente sul sito web dell'Accademia della Crusca (http://www.accademiadellacrusca.it/it/lingua-italiana/consulenza-linguistica); la rubrica Domande e risposte, presente sul portale Treccani - lingua italiana (http://www.treccani.it/lingua_italiana/domande_e_risposte/); e infine diverse pagine amatoriali pubblicate su social network come Facebook e dedicate alla salvaguardia della lingua italiana.

5. Ultima verifica 29 novembre 2017. 
la 'voce' dei parlanti. Il corpus di analisi è così costituito da una sezione di circa 69.000 parole formate dai commenti a due post di dubbi sull'italiano (i numeri 6 e $7^{6}$ ).

La scelta di lavorare su questa rubrica dipende soprattutto dal fatto che mentre nel caso dell'Accademia della Crusca i testi che rispondono ai quesiti dei lettori, pubblicati sulla rubrica Consulenza linguistica (685 risposte ad oggi ${ }^{7}$ ), non riportano la voce diretta degli utenti perché è la redazione che sintetizza le domande più frequenti e formula il quesito e la relativa risposta), invece nella rubrica di La Repubblica la voce dei lettori appare sia nei commenti sia nella formulazione dei dubbi linguistici. Il corpus di materiali è abbastanza ampio da consentire di individuare alcuni aspetti poco esplorati e poco discussi dalla linguistica ufficiale e che rivelano interessanti caratteristiche della linguistica ingenua.

\section{I risultati}

\subsection{Di che cosa si occupa la rubrica?}

La rubrica di Arcangeli pone problemi di varia natura che riguardano i vari livelli della lingua (dalla punteggiatura alla sintassi, dal lessico ad alcune questioni di pragmatica linguistica), ma che toccano anche visioni più ampie relative a norma e uso, oppure aspetti teorici della speculazione linguistica (ad esempio la contrapposizione semantica astratto - concreto), e che evidenziano, a loro volta, una certa visione della lingua dei redattori (si pensi alla titolazione dei post che può essere valutativa: ad esempio "S.O.S. Salviamo le nostre belle parole", "Gioventù digitalizzata"). I lettori possono intervenire - a proposito o a sproposito - con commenti che talvolta aggiungono dubbi e/o richieste di ulteriore chiarimento. Benché l'atteggiamento dei linguisti, il loro stile e l'ideologia di cui sono portatori sicuramente condizionano gli interventi degli utenti (anche rispetto alla terminologia, come si vedrà di seguito nell'esempio (31), per la polirematica testa del sintagma), in questa sede prescinderemo da tali elementi e variabili e, semplificando, lavoreremo soltanto sui commenti degli utenti esterni.

6. I due post Dubbi sull'italiano che si sono considerati sono pubblicati rispettivamente il 10/1/2014 e il 15/12/2015. II primo è seguito da 1105 commenti e il secondo da 509. I commenti si accumulano a partire dalla pubblicazione del post in avanti. Il Post Dubbi/6 riceve commenti dalla pubblicazione fino al febbraio 2017; il post Dubbi/7 riceve commenti fino al marzo 2017. Il corpus però qui utilizzato riguarda commenti pubblicati fino al dicembre 2015.

7. Ultima verifica 29 novembre 2017 cfr. http://www.accademiadellacrusca.it/it/lingua-italiana/consulenza-linguistica/domande-risposte. Va aggiunto peraltro che anche la Crusca ha istituito una consulenza su Social Network che invece rappresenta bene la voce degli utenti. Sulle consulenze linguistiche della Crusca si rimanda a Setti 2013 e Biffi e Setti 2013. 


\subsection{Quale terminologia usano gli utenti?}

L'analisi della terminologia settoriale utilizzata dagli utenti, che può essere più o meno debitrice della tradizione grammaticale o più o meno debitrice di quella della moderna linguistica, si può correlare al tipo di formazione degli utenti e può dare un'idea del tipo di discorsi che essi portano avanti nel blog. Pertanto l'analisi dei lemmi utilizzati riveste una particolare importanza.

A tal fine si è estratta la lista di frequenza delle parole utilizzate nel corpus (da un totale di 69.000 parole) dalla quale sono poi stati selezionati i termini del dominio linguistico (cioè relative a termini grammaticali e dell'attività metalinguistica, quindi anche lessemi di tipo valutativo come corretto, accettabile, giusto, sbagliato, ecc.).

La prima parola del dominio linguistico appare al $29^{\circ}$ posto ed è il lemma frase. Unendo le forme flesse di uno stesso lessema si ottiene una lista di 127 parole (cfr. Tabella 1) che vengono proposte in ordine decrescente rispetto al numero di occorrenze. Si sono considerati i lemmi che abbiano almeno 10 occorrenze: 
Tabella 1: Lista di parole ordinate per frequenza e appartenenti al dominio linguistico

\begin{tabular}{|c|c|c|}
\hline Frase/frasi 436 & Termine /termini 47 & Apposizione/apposizioni 22 \\
\hline $\begin{array}{c}\text { Complemento/complementi } \\
315\end{array}$ & Logica 46 & Comune 21 \\
\hline Corretto/corretta/corrette 251 & Funzione 46 & Costrutto/costrutti 21 \\
\hline Verbo/verbi 250 & Plurale/plurali 46 & Indefinito/indefiniti 20 \\
\hline Nome/ nomi 129 & Grammatica/grammatiche 45 & Maiuscola/maiuscolo 20 \\
\hline Uso/usi 123 & Condizionale /condizionali 43 & Accettabile/accettabili 20 \\
\hline Modo/modi 121 & Scritto 42 & $\begin{array}{c}\text { Semantico/semantica/seman- } \\
\text { tiche } 20\end{array}$ \\
\hline Analisi 119 & Contesto/contesti 41 & Accordo 19 \\
\hline Aggettivo/aggettivi 117 & Indiretta/indirette/indiretto 41 & Limitazione 19 \\
\hline Soggetto/soggetti 115 & Enunciato/enunciati 40 & Errore/errori 19 \\
\hline Dubbio/dubbi 114 & Azione 40 & Mezzo 18 \\
\hline Forma /forme 111 & Indicativo 40 & Qualità 18 \\
\hline Proposizione/proposizioni 106 & Genere/generi 39 & Causale 17 \\
\hline Predicato/predicati 101 & Temporale/temporali 39 & Fraseologico 17 \\
\hline Subordinata/subordinate 100 & Participio 37 & Parlato 17 \\
\hline Congiuntivo 100 & Sostantivo/sostantivi 37 & Attributo/attributi 17 \\
\hline Espressione/espressioni 96 & Implicita /implicite 36 & Impersonale/impersonali 17 \\
\hline Preposizione/ preposizioni 93 & Maschile/maschili 32 & Figurato /figurati 16 \\
\hline Parola/parole 92 & Regola/regole 32 & Paragone 16 \\
\hline Avverbio/avverbi 88 & Congiunzione/congiunzioni 32 & Numerale/numerali 16 \\
\hline Relativa/relative/relativo 88 & Avverbiale/avverbiali 31 & Assoluto/assoluta/assoluti 16 \\
\hline Luogo 79 & Singolare/singolari 31 & Limitativa/limitative 16 \\
\hline Tempo /tempi 79 & Coordinata/coordinate 30 & Discorso 15 \\
\hline Persona/persone 79 & Comparativo 30 & Composto/composti 15 \\
\hline Passato 78 & Numero 29 & Origine 15 \\
\hline Punto/punti 73 & Futuro 29 & Diretto 14 \\
\hline Verbale /verbali 71 & Reggente 28 & Minuscola 14 \\
\hline Principale/ principali 70 & Infinito/infiniti 28 & Concessiva/concessive 14 \\
\hline Oggetto 68 & Nominale/nominali 27 & Comparativa/comparative 14 \\
\hline Presente 67 & Testo/testi 26 & Argomento 13 \\
\hline
\end{tabular}




\begin{tabular}{|c|c|c|}
\hline Oggettiva /oggettive 63 & Causa 25 & Trapassato/trapassati 13 \\
\hline Periodo/periodi 60 & Costruzione/costruzioni 25 & Copula 12 \\
\hline Parte 59 & Femminile/femminili 25 12 \\
\hline Grammaticale/grammaticali & Specificazione 24 & Aggettivale/aggettivali 12 \\
\hline Significato/significati 57 & $\begin{array}{c}\text { Sbagliato/sbagliata/sbagliate/ } \\
\text { sbagliati 24 }\end{array}$ & Logico 12 \\
\hline Senso/sensi 57 & Pronominale/pronominali 24 & Voce 12 \\
\hline Giusto/giusta/giuste 56 & Predicativo/predicativi 23 & Esplicita/esplicite 11 \\
\hline Locuzione/locuzioni 53 & Finale/finali 23 & Modale/modali 11 \\
\hline Virgola/virgole 52 & Imperfetto 23 & Aspetto 10 \\
\hline Articolo/articoli 52 & Sintagma 22 & Gerundio 10 \\
\hline Interrogativa/interrogative/in- & Fine 22 & \\
\hline terrogativo 52 & Quantità 22 & \\
\hline Superlativo/superlativi 49 & Categoria/categorie 22 & \\
\hline Grado/gradi 49 & & \\
\hline
\end{tabular}

Occorre fare subito alcune precisazioni. Innanzitutto è chiaro che stiamo operando vari tipi di semplificazioni nell'utilizzare la lista delle parole per ricostruire i discorsi degli utenti, e questo non solo perché la lista si riferisce a tutto lo scambio contenuto nel corpus, e quindi include anche gli interventi dei linguisti, ma soprattutto perché alcuni termini tecnici della linguistica possono anche ricorrere come parole comuni (esempio parole come modo, parte, punto) oppure hanno più significati tecnici: indiretto si riferisce sia a discorso (discorso indiretto) sia a complemento (complemento indiretto), sia a oggetto (oggetto indiretto).

Inoltre la lista così come presentata include sia tecnicismi (soggetto, superlativo, proposizione, preposizione), sia espressioni valutative che gli utenti sviluppano nei loro interventi (dubbio, corretto, uso, contesto, giusto, accettabile).

A questo punto è stato utile suddividere ulteriormente la lista separando i termini tecnici da quelli valutativi. Rispetto ai primi sarà utile osservare alcune 'assenze' e infine provare a assegnare i lessemi ai vari livelli dell'analisi linguistica. Nella Tabella 2 si ripropone la lista della Tabella 1, suddivisa per livelli dell'analisi linguistica8:

8. L'attribuzione dei lemmi ai livelli d'analisi è in qualche caso arbitraria: ad esempio l'accordo è un termine sia sintattico sia morfologico, ma si è preferito di non annotare più volte uno stesso lemma. 
Tabella 2 Distribuzione delle parole del dominio linguistico in base ai livelli di analisi linguistica pertinenti

\begin{tabular}{|c|c|c|c|c|}
\hline Ortografia & Sintassi & Morfologia & $\begin{array}{c}\text { Lessico e se- } \\
\text { mantica }\end{array}$ & $\begin{array}{l}\text { Valutazione e } \\
\text { metalinguaggio }\end{array}$ \\
\hline Virgola & Accordo & Avverbiale & Fraseologico & Testo \\
\hline Punto & Apposizione & Nominale & Comune & Accettabile \\
\hline Minuscola & Argomento & Trapassato & Figurato & Uso \\
\hline \multirow[t]{30}{*}{ Maiuscola } & Aspetto & Aggettivale & Termine & Costrutto \\
\hline & Attributo & Quantità & Significato & Discorso \\
\hline & Azione & Locuzione & Senso & Corretto \\
\hline & Causa & Indicativo & Semantica & Parte \\
\hline & Causale & Comune & & Sintagma \\
\hline & Comparativa & Composto & & Grammatica \\
\hline & Complemento & Nome & & Grammaticale \\
\hline & Concessiva & Numerale & & Giusto \\
\hline & Coordinata & Genere & & Parlato \\
\hline & Copula & Remoto & & Scritto \\
\hline & Diretto & Interrogativo & & Contesto \\
\hline & Enunciato & Plurale & & Espressione \\
\hline & Esplicita & Gerundio & & Dubbio \\
\hline & Finale & Numero & & Categoria \\
\hline & Fine & Singolare & & Regola \\
\hline & Frase & Verbo & & Logica \\
\hline & Funzione & Verbale & & Logico \\
\hline & Impersonale & Comune & & Analisi \\
\hline & Implicita & Grado & & Sbagliato \\
\hline & Indiretta & Femminile & & Costruzione \\
\hline & Indiretto & Qualità & & \\
\hline & Interrogativa & Futuro & & \\
\hline & Limitativa & Infinito & & \\
\hline & Limitazione & Voce & & \\
\hline & Luogo & Congiunzione & & \\
\hline & Mezzo & Parola & & \\
\hline & Modo & Imperfetto & & \\
\hline & Nominale & Congiuntivo & & \\
\hline & Oggettiva & Comparativo & & \\
\hline & Oggetto & Tempo & & \\
\hline
\end{tabular}




\begin{tabular}{|c|c|c|c|c|}
\hline Ortografia & Sintassi & Morfologia & $\begin{array}{c}\text { Lessico e se- } \\
\text { mantica }\end{array}$ & $\begin{array}{c}\text { Valutazione e } \\
\text { metalinguaggio }\end{array}$ \\
\hline & Origine & Persona & & \\
\hline & Paragone & Indefinito & & \\
\hline & Periodo & Pronominale & & \\
\hline & Predicativo & Presente & & \\
\hline & Predicato & Sostantivo & & \\
\hline & Principale & Avverbio & & \\
\hline & Proposizione & Passato & & \\
\hline & Reggente & Forma & & \\
\hline & Relativa & Condizionale & & \\
\hline & Soggetto & Superlativo & & \\
\hline & Specificazione & Preposizione & & \\
\hline & Subordinata & Participio & & \\
\hline & Temporale & Aggettivo & & \\
\hline & Termine & Articolo & & \\
\hline & Testa & Relativo & & \\
\hline & Verbale & Modale & & \\
\hline & & Maschile & & \\
\hline & & Assoluto & & \\
\hline
\end{tabular}

Osserviamo innanzitutto che mancano alcuni tecnicismi generali che designano i livelli dell'analisi linguistica, e cioè ortografia, sintassi, morfologia, fonologia, fonetica, lessico. Si trovano invece la parola grammatica (45 occorrenze), i termini semantica - semantico (20 occorrenze), punteggiatura (8 occorrenze) e si fa riferimento spesso all'analisi logica (40 occorrenze) o all'analisi grammaticale (21 occorrenze), o infine anche all'analisi del periodo (26 occorrenze), cioè a pratiche analitiche della tradizione scolastica:

(2) Buongiorno, le chiedo gentilmente l'analisi logica della seguente frase: "In Italia, nei secoli precedenti l'Ottocento, era stato praticato il gioco della palla". Grazie.

I quesiti che sottopongono ipotesi di analisi logica da far valutare agli esperti sono molteplici e tradiscono più che un'esigenza di correttezza linguistica un'ansia da «prestazione» del tipo incoraggiato e praticato nel contesto scolastico. Se ne veda un esempio nell'estratto (3):

(3) Due ultime domande sulla frase "Ho mangiato della frutta: due mele, tre pere, quattro banane": [...] e come vanno classificati in analisi logica? Grazie ancora.

Il termine semantico però può anche ricorrere in modo non tecnico, come banale sinonimo di significato, solo per impreziosire la scrittura, come nell'esempio che segue: 
(4) 11 marzo 2014 alle 23:01 Nella frase :"Frederic vede una guardia scagliarsi con la spada in pugno, contro un insorto, che cade morto". Vi chiedo se la seconda virgola sia corretta. Il pronome relativo "che" non si lega direttamente al nome "insorto"? potreste gentilmente spiegarmi se le virgole siano proprio necessarie, dal momento che dividono precise unità semantiche. Grazie

Andando per livelli, osserviamo che si parla abbastanza poco di grafia e di segni d'interpunzione. La parola punto infatti ricorre solo 6-7 volte per designare il segno di interpunzione (in collocazione con l'aggettivo fermo). La virgola viene evocata in qualche risposta per sanzionare la separazione tra soggetto e predicato. Più spesso i quesiti riferiti al livello grafico riguardano l'uso delle maiuscole:

(5) Gentile professore, se scrivo "la capitale" riferendomi a Roma, devo usare la C maiuscola?

E se scrivo "la capitale tedesca"?

La maggior parte dei termini (e dei quesiti) rientra nell'area della sintassi e della morfologia, come si può ricavare considerando l'alto numero di termini appartenenti a queste due aree.

Per quanto concerne la morfologia appaiono tutti i termini che designano le classi di parole ad eccezione delle interiezioni (nome, verbo, aggettivo, pronome, articolo, avverbio, congiunzione, preposizione) e sono attestati gli aggettivi corrispondenti (nominale, verbale, aggettivale, pronominale, avverbiale). Ricorrono poi termini che designano categorie morfologiche (genere, numero, persona) e i possibili valori che tali categorie assumono in italiano (maschile, femminile, singolare, plurale). Si trovano anche i termini che designano modi e tempi verbali (passato remoto, trapassato, presente, futuro, imperfetto, indicativo, congiuntivo, condizionale, participio, gerundio, infinito) o sottocategorie nell'ambito delle classi di parole (numerale, interrogativo, superlativo relativo e assoluto, grado, comparativo, indefinito). Frequenti infine i lemmi quantità (che ricorre una ventina di volte perlopiù in unione con avverbio o complemento) e qualità (quest'ultimo con una gamma maggiore di accezioni, non solo usato per discutere di aggettivi che 'implicano la qualità di' o del complemento di qualità, ma in molti casi la parola ricorre nella perifrasi in qualità di). Infine registriamo alcuni lemmi che rimandano all'analisi grammaticale classica (comune, riferito a nome) o alla formazione delle parole (composto). La presenza della parola aspetto non deve alimentare illusioni: come termine specialistico è poco noto, e nel corpus non ricorre mai nell'accezione linguistica.

Le ultime tre parole dell'ambito morfologico riguardano la designazione delle unità stesse della morfologia: forma, locuzione e voce. L'ultimo termine occorre quasi sempre nella espressione voce del verbo ... (o poi designa la voce umana), quindi ricorre nel contesto tradizionalissimo dell'analisi delle forme verbali (amo, voce del verbo amare, prima persona ecc.). Il termine forma ha usi molteplici e vale spesso come sinonimo di variante (forma bassa colloquiale, forma settentrionale). Infine il termine locuzione ricorre 53 volte, spesso in unione coll'aggettivo avverbiale, per designare un'ampia gamma di espressioni polirematiche sulla cui analisi morfologica o sulle cui funzioni sintattiche si orientano molti quesiti, a volte con un accanimento analitico come nell'esempio (6): 
(6) Egregio dott. Aresti, la ringrazio per la risposta. Mi resta un dubbio: in "Il cinquanta per cento degli italiani va in vacanza", "per cento" può considerarsi una locuzione avverbiale distributiva? Grazie ancora

Nell'ambito della terminologia sintattica si rileva un gruppo di lessemi che si riferisce al livello dei sintagmi e cioè termini che indicano gli elementi e le funzioni principali all'interno della frase (sintagma, complemento, apposizione, attributo, soggetto, oggetto, predicato verbale e nominale, copula). Poi un gruppo di termini che designa i singoli complementi esplorati dall'analisi logica più tradizionale (causa, fine, diretto, indiretto, limitazione, luogo mezzo, origine, paragone, modo, termine, specificazione, predicativo).

A livello di frase complessa sono presenti i termini generali (coordinata, subordinata, implicita, esplicita, principale, reggente) e poi i termini che si riferiscono a tipi di frasi subordinate (comparativa, concessiva, finale, interrogativa, causale, limitativa, oggettiva, temporale, relativa).

Per quanto riguarda la definizione delle unità linguistiche del livello sintattico ricorrono le tradizionali frase, proposizione, periodo, ma anche i più moderni sintagma (22 occorrenze) e enunciato, un termine dotato di maggiore estensione, utilizzabile per riferirsi a porzioni autonome di testi o discorsi, riferibile quindi tanto all'orale quanto allo scritto. Un esempio abbastanza rigoroso dell'uso del termine sintagma è il seguente:

(7) Un saluto a tutti, nelle frasi: "La colpa di tutto siete voi", "La prospettiva più grave sarebbero i problemi economici" quali sintagmi fungono da soggetto (io propenderei per "voi" e "i problemi economici")? Grazie

ma in genere il termine ricorre nei post della redazione. Anche per il termine enunciato accade qualcosa di analogo: delle 40 occorrenze solo due sono i casi in cui la parola è usata da un lettore:

(8) 12 luglio 2014 alle 10:51 Buongiorno, eccomi al secondo quesito, che riguarda l'articolo nelle enumerazioni. Ho letto che di solito si omette in questi casi, ma è una regola ferrea che è bene osservare oppure è solo un consiglio?

1. Frutta, verdura e pasta sono i cibi che fanno bene alla salute.

2. Frutta, verdura e pasta sono cibi che fanno bene alla salute.

3. La frutta, la verdura e la pasta sono cibi che fanno bene alla salute.

4. La frutta, la verdura e la pasta sono i cibi che fanno bene alla salute.

Omettendo l'articolo nel soggetto andrà omesso anche nel complemento? Quali sono le frasi più corrette in uno scritto? Grazie, perdonatemi del lungo enunciato. 
Si rilevano anche i termini costrutto, costruzione, espressione che designano unità linguistiche in modo generico, le prime due parole sono però quasi solo usate dai linguisti della redazione. Costruzione ricorre non solo come sinonimo di costrutto, e quindi con valore di iperonimo rispetto a diverse entità linguistiche (complemento, frase, proposizione), ma ricorre anche come nome di azione vero e proprio, a conferma del suo non essere stabilizzato come termine tecnico:

(9) 2 aprile 2014 alle 15:04 Gentile professore, in merito alla mia domanda precedente, cambia qualcosa se la costruzione della frase diventa questa? [...]

Altre quattro parole dell'ambito sintattico sono particolarmente interessanti e significative per dare conto dell'ideologia linguistica e della linguistica ingenua degli utenti. Si tratta dei termini azione (40 occorrenze), accordo (19 occorrenze), funzione (46 occorrenze) e testa (12 occorrenze), analizzati nel paragrafo 4.4.

Per quanto riguarda l'ambito del lessico si osservino le parole termine (che però vale anche per complemento di termine o per secondo termine di paragone), l'aggettivo comune (nella collocazione 'nome comune di cosa o persona', diffusa a partire dall'analisi grammaticale scolastica), usato come opposto di (nome) proprio, oppure nel significato di uso frequente, in contrapposizione a raro o non ricercato (rientra quindi nel gruppo dei termini valutativi):

(10) 16 settembre 2013 alle 18:40 Salve, vi pongo un quesito nato da una discussione tra amici. Qual'è (sic!) il significato del termine PANDERE ? può essere usato come sinonimo di rivelare, svelare?

(11) 16 dicembre 2009 alle 12:42 Nei dizionari "corredato" regge sempre la preposizione "di", ma nell'uso comune e nel parlato sono utilizzate ed accettate entrambe le preposizioni ("di" e "da").

Gli altri tre termini inseriti in questo livello sono significato (57 occorrenze), senso (57 occorrenze) e figurato (16 occorrenze). Si tratta di termini frequenti e usati opportunamente cioè come tecnicismi.

Per i termini valutativi si rimanda al paragrafo 4.5.

Accanto alla quasi totale assenza, ad eccezione di semantica e grammatica, di termini designanti i livelli dell'analisi linguistica (fonetica, fonologia, morfologia, sintassi, pragmatica), è degna di nota l'assenza di alcune altre parole pregnanti nel dominio linguistico. In ambito sintattico non si registrano parole che rimandano ad approcci meno tradizionali (come dipendente, circostanziale, valenza, clausola). Il termine argomento, che si usa in linguistica per individuare i costituenti della valenza di un verbo (da cui l'aggettivo struttura argomentale), ricorre nel corpus o per indicare il complemento di argomento, oppure nell'accezione comune (un saggio sull'argomento, che trova qui). Scarsa la presenza di completiva (ricorre perlopiù nei post della redazione). Anche per la morfologia, oltre a segnalarsi l'assenza della parola polirematica, si osserva l'assenza di prefisso e suffisso così come di 
connettivo, determinante, derivato, primitivo, alterato. In generale quindi la terminologia ricorrente è ampia ma molto tradizionale.

\subsection{Quali problemi e dubbi sono più ricorrenti?}

La maggior parte dei quesiti propone esempi linguistici su cui si chiedono pareri a) di correttezza - accettabilità rispetto alla norma (meno spesso ci sono riferimenti all'uso linguistico); b) di appropriatezza dell'analisi linguistica: si chiede cioè al linguista di fare l'analisi - grammaticale, logica, del periodo - di esempi oppure di dare un parere sulla correttezza dell'analisi proposta dal lettore.

Nelle richieste di valutazione della correttezza di esempi linguistici proposti dai lettori, spesso gli utenti tradiscono una visione della lingua più rigida di quella del linguista9. L'utente chiede regole nette - corretto /sbagliato - e spesso non considera che ci possano essere differenze d'uso legate al contesto, o al canale (discussioni su scritto e parlato, ad esempio mancano tra i lettori, anche se vi fanno riferimento i redattori parlando ad esempio di scritto formale di parlato colloquiale) o semplicemente alla variabilità linguistica. In generale la preoccupazione degli utenti è orientata sulla correttezza della lingua scritta:

(12) 18 settembre 2013 alle 17:49 Mi scusi, dimenticavo altre due richieste: 1) "ho detto loro" (in caso di più persone) so che è corretto, ma alcune colleghe accettano in questo caso anche "gli ho detto" senza correggerlo in un testo scritto. Come mi regolo

(13) Omettendo l'articolo nel soggetto andrà omesso anche nel complemento? Quali sono le frasi più corrette in uno scritto? Grazie, perdonatemi del lungo enunciato.

Il caso molto frequente di dubbi linguistici che richiedono alla redazione valutazioni di analisi e classificazioni grammaticali proposte dai lettori conferma il prosperare di una certa tradizione della didattica della lingua e della grammatica. I lettori sembrano coscienti del peso della tradizione grammaticale [cfr. esempio (14)] e non utilizzano spesso i concetti della linguistica moderna [con qualche eccezione, come ad esempio il caso dell'esempio (15)]:

(14) Buongiorno, mi rendo conto che le manie di classificazione in analisi logica siano talvolta inutili, se non fuorvianti, ma non posso fare a meno di chiedermi: [...]

(15) 22 ottobre 2013 alle 16:54 Buonasera, ho qualche problema con le denominazioni delle strutture marcate di focalizzazione. Se non erro "E' questo che funzionerà" è una scissa, "Quello che voglio è che funzioni” è una pseudoscissa, [...]

9. Sulla norma linguistica cfr. almeno Antonelli 2007, D’Achille 2014, Serianni 2006, 2007. 
Quanto alla formulazione dei quesiti, c'è molta variabilità nella struttura, ma domina mediamente un tono rispettoso, gentile e in parte anche semplice, tipico del contesto (social network). Gli scriventi infatti si rivolgono a volte in modo diretto ai redattori, integrano le risposte degli stessi, salutano, ringraziano, fanno complimenti e inviano auguri di buona giornata. E nel fare le richieste più varie con molta semplicità mostrano un rapporto di fiducia e di interesse nei confronti del blog:

(16) 11 dicembre 2013 alle 16:09 Buongiorno, vorrei sapere se l'interiezione "Madonna!" (con le sue varianti "oh Madonna!", "Madonna mia!" ecc.) ha una connotazione negativa/offensiva o se oramai può essere considerata come equivalente di "Mammamia!". Grazie

\subsection{Quali concetti ingenui vengono utilizzati dagli utenti?}

Per iniziare a dare conto della 'linguistica ingenua' dei lettori si osservino le quattro parole dell'ambito sintattico particolarmente interessanti e significative. Si tratta dei termini accordo (19 occorrenze), azione (40 occorrenze), funzione (46 occorrenze) e testa (12 occorrenze).

La parola accordo appare sia nell'espressione essere d'accordo sia nel significato specialistico di principio sintattico che opera nella lingua italiana mediante strumenti morfologici per segnalare coesione tra elementi (accordo dell'aggettivo col nome, del verbo col soggetto). Questa seconda accezione è però minoritaria nel corpus. Ci sono alcuni casi in cui il dubbio linguistico verte proprio sull'accordo (di genere, numero o del participio). Vediamo due esempi qui riportati come (17) e (18):

(17) 22 maggio 2015 alle 11:04 Salve, ho un dubbio su un accordo di genere. In una frase tipo: "Sono stat* pubblicat* 100 milioni di copie della brochure e 50 milioni di altre pubblicazioni", l'accordo va al maschile o al femminile? Dalle mie ricerche mi pare di poter concludere che ormai entrambi gli accordi sono accettati, ma qual è il più corretto dal punto di vista grammaticale? Grazie molte.

(18) 9 luglio 2015 alle 11:42 Buongiorno, si dice "ne ho comprati due chili" oppure "ne ho comprate due chili" quando per es. racconto di essere andata dal fruttivendolo e ho comprato 2 chili di mele. L'accordo è sulla quantità o sull'oggetto? Mille grazie!

Benché la parola accordo sia presente in questa accezione tecnica, l'uso tradisce una percezione superficiale e parziale del fenomeno, come è anche prevedibile che sia. Come si può osservare in (17) lo scrivente definisce in modo corretto il fenomeno di accordo di genere, ma gli manca la comprensione generale del problema, e cioè il fatto che l'accordo è un principio sintattico che riguarda la relazione tra soggetto e participio passato in cui le ricadute morfologiche sono solo l'effetto visibile del principio sintattico, effetto che può risultare distorto nel caso in cui il soggetto sia costituito da un sintagma complesso con un sintagma nominale che indica una unità di misura seguita da un complemento di specificazione. Lo stesso accade nel post riportato in (18) dove il tema dell'accordo del participio riguarda la possibilità di accordo col partitivo (ne) o col complemento oggetto. 
La parola azione è un termine chiave della grammatica tradizionale utilizzato per riferirsi all'elemento verbale di una frase: l'azione costituirebbe il tipico designatum di un verbo (posizione superata dalla linguistica, come è noto, dove si parla più spesso di eventi, stati, condizioni, ma poco recepita dalla grammatica tradizionale). Il termine ricorre nel corpus spesso nell'espressione compiere l'azione/l'azione compiuta, indicata dal verbo $(19,20)$ :

(19) [...] Il complemento di scopo o fine indica il motivo (fine) per cui si compie l'azione: combattiamo "per il riconoscimento" dei nostri diritti. [...]

(20) salve, è corretto dire: il modo gerundio indica un'azione contemporanea ad un'altra che si svolge ed è con essa in rapporto di CAUSA/MODO/TEMPO/MEZZO? [...]

Infine per quanto riguarda la parola funzione essa ricorre con un'accezione comune (in funzione di) e con accezione tecnica legata soprattutto all'analisi logica (funzione di soggetto/oggetto) oppure ricorre per definire il caso di categorie morfologiche (come ad esempio l'avverbio prima) che entrando in locuzioni con preposizioni (prima di) vengono usate 'in funzione di' altre categorie (nella fattispecie la locuzione avverbiale è usata con la funzione di una preposizione: prima di). Anche in questo caso comunque la parola ricorre più spesso nei post della redazione che in quelli degli utenti:

(21) 19 aprile 2015 alle 12:54 Buongiorno, vi domando in quali delle seguenti frasi ed espressioni "niente" ricopre funzione pronominale e in quali una funzione avverbiale [...]

(22) 19 maggio 2014 alle 10:32 Abbiamo due periodi. $1^{\circ}$ Si sa la gente doveva lavorare duramente. $2^{\circ}$ Si dice anche che le popolazioni della Lombardia preparassero un tipo di pane simile alla piadina. La questione é (sic!) la seguente: i due SI che funzione hanno: impersonale o passiva? E hanno la stessa funzione? Mi trovo in difficoltà poiché mi sembrano entrambi aventi la stessa funzione, istintivamente direi impersonale, ma guardando attentamente il periodo $[\ldots]$

Un caso interessante è rappresentato dal termine testa che mostra un esempio di 'apprendimento del linguaggio specialistico'. Anche questo lemma ricorre 12 volte e solo 4 volte col valore specialistico di 'elemento indispensabile e perno di un sintagma'. Lo usa tre volte Arcangeli e solo una volta un utente. Uno dei casi in cui Arcangeli usa la parola testa ricorre nel commentare e correggere il dubbio linguistico di Rita (in relazione al sintagma numero venti), subito dopo un altro utente lo riutilizza, con evidente intento imitativo riallacciandosi al quesito di Rita. Curiosamente Arcangeli nella replica elimina la parola testa e introduce l'espressione parola guida. Si riportano in (23) i 4 testi in cui si sviluppa il dialogo che abbiamo appena riassunto: 
(23) 22 marzo 2015 alle 20:40 Buonasera. Vorrei chiedere: nell'espressione "il numero venti” la parola "venti" potrebbe essere nome e "numero" apposizione? Grazie, Rita

linguista_1 scrive: 22 marzo 2015 alle 22:58 Esattamente il contrario. La testa del sintagma è "numero", l'elemento aggiuntivo (che ha dunque il valore di un'apposizione) è "venti". Massimo Arcangeli

3 aprile 2015 alle 19:58 Buonasera, desidererei alcune delucidazioni: [...] 2) mi riallaccio alla domanda di Rita sul "numero venti": come riconoscere la testa del sintagma dall'apposizione? [...]

linguista_1 scrive: 5 aprile 2015 alle 11:55 [... 2) La parola guida del sintagma è "numero", non "venti" (che fa da semplice accessorio). [...] Massimo Arcangeli

Infine nell'esempio (24) si condensano diversi aspetti significativi in merito alla visione 'ingenua' dei parlanti sul funzionamento della lingua e sul rapporto tra sostanza fonica e convenzioni grafiche. In particolare l'autore del post esprime vari dubbi sull'uso del punto interrogativo nelle interrogative indirette e rispetto ad altre convenzioni grafiche (uso delle parentesi):

(24) 17 settembre 2013 alle 18:05 USO DEL PUNTO INTERROGATIVO. Buon giorno agli amici del linguista. Qualche giorno fa ho avuto da dire sul corretto uso del punto interrogativo. In pratica, io sostengo che un'eventuale supplemento di info tra parentesi deve essere messo prima della chiusura. 1) Pensi che sia davvero così povero (non è una domanda retorica)? Dunque, per me va bene l'esempio 1. Mentre lui sostiene il contrario: 2) Pensi che sia davvero così povero? (Non è una domanda retorica) Poi, un'altra regola che ho trovato è quella che il PI non si usa in caso di indirette. L'esempio che ho trovato è questo: "Chissà se posso chiederle un favore", senza punto. Però, a livello di suono, per me ci andrebbe, non so se riusciate a capirmi. (Chissà se posso chiederle un favore?) sarebbe come "Posso chiederle un favore, chissà?" Forse se cambio in "Chiederti" è diverso? Grazie. Un saluto

Un altro utente percepisce la possibilità e necessità di separare i piani dell'analisi, in questo caso il livello delle funzioni sintattiche e l'aspetto pragmatico, ma rimane legato all'unico approccio che gli offre la formazione tradizionale, cioè quello dell'analisi logica:

(25) Nell'analisi logica, come si può interpretare un sostantivo inserito in questo modo: "guai se vieni in ritardo". "guai" è un complemento oggetto, anche se assume un valore di avvertimento? Grazie

Il seguente post infine mostra l'acume e la sensibilità dell'utente rispetto al parametro dell'agentività (che sta alla base della differenza semantica tra agente e causa) affrontato però con un linguaggio non specialistico: 
(26) 3 novembre 2014 alle 22:16 Un saluto a tutti, ecco alcune domande da sottoporvi: 1) "Fu applaudito a lungo dal pubblico" e "Fu apprezzato dalla parrocchia": siamo in presenza di complementi d'agente o di causa efficiente? 2) Gli animali ricadono nella categoria dei complementi d'agente o di causa efficiente? 3) "Non so quanto spendere": "quanto" è avverbio interrogativo? Se sì, si può affermare che è usato in funzione di congiunzione? Grazie

\subsection{Ideologia linguistica}

La possibilità di tracciare l'ideologia linguistica dei lettori della rubrica si basa su alcune considerazioni già introdotte nel paragrafo 4.3, e cioè sul fatto che i lettori cercano risposte nette - in termini di giusto/corretto vs. sbagliato - ai loro dubbi linguistici. Non ci sono quasi mai posizioni sfumate in relazione a contesti d'uso, a variabilità diamesica (scritto - orale), o rispetto ad altre dimensioni della variazione linguistica ${ }^{10}$. Non si rileva traccia del dibattito di matrice chomskiana sull'accettabilità o grammaticalità degli enunciati. L'aggettivo grammaticale, quando appare, e perlopiù nei post della redazione, ricorre comunque riferito a forma, tradizione, analisi e non ha il significato tecnico di 'espressione ben formata'. Quindi gli utenti vedono la lingua in termini normativi tradizionali e la grammatica come il suo sistema regolativo. In un approccio di questo tipo, cioè normativo, si spiega bene la presenza dei termini regola e errore. La regola viene spesso invocata (c'è una regola?) di fronte al dubbio e alla molteplicità di soluzioni, è una regola ferrea quella a cui pensa il lettore, che possa guidare e tranquillizzare il parlante e fornire spiegazioni:

(27) 23 settembre 2013 alle 08:40 Gentile Linguista, si dice "accompagnare qualcuno alla/in stazione”? E qual è la regola/spiegazione a proposito? Grazie!

(28) 5 marzo 2014 alle 14:30 Gentile Dott. Aresti, grazie per la Sua cortese risposta. [...] È unıabitudine o c’è una regola? La ringrazio in anticipo.

(29) 12 luglio 2014 alle 10:51 Buongiorno, eccomi al secondo quesito, [...] ma è una regola ferrea che è bene osservare oppure è solo un consiglio?

In un quadro di incertezza normativa, l'errore è sempre 'in agguato'. Nell'esempio (30) l'utente lo descrive quasi come una colpa di tipo morale-religioso: si parla di «grave errore» (sembra di sentire echeggiare il concetto di 'peccato capitale', di matrice religiosa) di lingua italiana:

10. Una visione analoga emerge nello studio di Tassone (2015) a proposito dell'ideologia linguistica dei lettori che scrivono agli esperti dell'Accademia della Crusca, e le cui domande appaiono nel foglio La Crusca per voi: «ll foglio continua ad ospitare richieste che si collocano ai poli dicotomici del giusto/sbagliato» (Tassone 2015: 149). 
(30) 11 giugno 2014 alle 10:31 Gentile Professore, avrei un dubbio sull'uso dell'aggettivo "difettato". Alcuni dizionari lo registrano ancora seppur consigliando l'uso del più comune "difettoso", altri invece presentano solo quest'ultimo. Vorrei sapere, usando il primo, commetto un grave errore di lingua italiana? La ringrazio per l'attenzione e complimenti per il forum interamente dedicato alla nostra lingua.

L'errore ripetuto, diffuso e non estirpato, secondo l'utente genera l'abitudine e l'accettabilità. Ma anche questa evoluzione viene dipinta come una sorta di 'decadimento dei costumi'. Talvolta ci si accorge che gli esempi proposti e sottoposti al giudizio dell'esperto non costituiscono una violazione della regola invocata, come si vede nella fine dell'estratto (31):

(31) 12 giugno 2015 alle 22:06 Egregi linguisti, le grammatiche italiane ricordano che è possibile esprimere la passività anche grazie ad ausiliari quali venire ed andare in unione con il participio passato. Le stesse, però, ricordano che [...] o son - molto più semplicemente - errori a cui alcuni di noi si stanno, purtroppo, abituando. Vi ringrazio in anticipo per il prezioso contributo e per la Vostra disponibilità.

Vivacizza il quadro fin qui proposto, che denota una visione dicotomica bianco/nero, la presenza dell'aggettivo accettabile (14 occorrenze), che però è usato in 10 casi dalla redazione, e solo in 4 dagli utenti. Si vedano a questo riguardo i due post seguenti:

(32) 16 gennaio 2014 alle 10:47 Vorrei qualche chiarimento sull'uso del femminile per nomi riferiti a professioni che tradizionalmente non erano svolti dalle donne. [...] Il linguista trova accettabile: "il vigile Maria Rossi”. So che la situazione in questi casi è molto fluida; ma sarebbe possibile avere indicazioni più precise? Possibile che non esistano in merito norme chiare e codificate?

(33) 28 gennaio 2014 alle 22:58 Buonasera, vorrei sapere se nelle frasi seguenti il termine di paragone 'che' è accettabile come alternativa all'uso standard di 'di'. Grazie. [... ]

I post (32) e (33) mostrano bene in azione l'immaginario linguistico dei nostri parlanti. Nel primo si coglie infatti la percezione di una fluidità dei fatti di lingua, in certe aree della grammatica, che però non viene totalmente accettata, per cui ritorna la richiesta di una norma codificata e chiara. Invece innovativo è il post (33) in cui balena l'idea che si possa discutere di usi accettabili, che si contrappongono all'uso standard, e quindi la dicotomia bianco/nero sembra arricchirsi di qualche tonalità di grigio.

Di fatto però ragionamenti più articolati sulla complessità e variabilità della lingua non emergono: sono del tutto assenti termini come standard, substandard, o l'aggettivo normativo o si attestano al di sotto delle 10 occorrenze (per il termine norma le occorrenze sono 8 ma tra queste ci sono anche usi poco pregnanti come l'espressione di norma e 4-5 usi di norma grammaticale e norma ortogra- 
fica). Anche il termine variabilità manca e gli aggettivi variabile e invariabile sono usati soltanto in collocazione con parti del discorso, dunque in contesto morfologico.

Che la grammatica sia un'area in cui si sollevano dubbi è suggerito dallo stesso blog: per cui non stupisce che molti lettori inizino il loro commento evocando questo termine e collegandolo a un qualche fenomeno (ho un dubbio sulla seguente frase, ecc.).

Da alcuni post si ricava comunque che i parlanti sono sensibili a molti aspetti della lingua e a fatti linguistici che richiedono inquadramenti vari, ad esempio in riferimento alla variabilità diatopica (a usi regionali e alle varietà dialettali):

(34) Salve ho una richiesta un po' complessa. La locuzione "tanto bene" usata al posto di "neanche a farlo apposta" ovvero come "sequenza di accadimenti casuali", esiste in Italiano o è dialettale della mia rgione (Umbria)? Alcuni esempi: [...]

È anche interessante osservare come la parola contesto (circa 40 occorrenze) sia diffusa anche nella lingua dei non linguisti e supporta l'idea che le frasi e i discorsi possano avere una diversa accettabilità e validità in base al contesto d'uso inteso sia nel senso di cotesto, sia nel senso di ambito d'uso o addirittura evento comunicativo. I parlanti cioè talvolta cercano di contestualizzare i loro dubbi grammaticali perché sanno che è possibile ammettere una variazione in base al contesto (cfr. 35):

(35) 8 dicembre 2013 alle 11:25 Buongiorno, avrei un'altro dubbio grammaticale, il contesto e' il seguente: [...] Nel contesto che ho fornito qual e' la frase corretta secondo lei?

Per concludere si consideri un ultimo post in cui il concetto di contesto come fattore che può interferire sulla scelta tra varianti sintattiche è «declinato» in una maniera sicuramente singolare:

(36) 28 ottobre 2013 alle 10:15 Salve, ho un dubbio riguardo la costruzione di un frase:

- non credevo potesse mancarmi cosi (sic!) tanto...

- non credevo mi sarebbe mancata cosi (sic!) tanto...

quale delle due e' (sic!) corretta? il contesto riguarda una persona con cui ho interrotto i rapporti. grazie 


\section{Conclusioni}

L'analisi della rubrica linguistica ha consentito di evidenziare diversi aspetti rilevanti rispetto agli obiettivi proposti. Innanzitutto si è ricostruito un identikit del 'linguista ingenuo' e si è potuta tracciare la sua idea di lingua. Il linguista ingenuo è preoccupato e solleva dubbi soprattutto in merito a questioni morfologiche e sintattiche, secondo scelte tematiche abbastanza vicine alla tradizione scolastica. Talvolta sottopone all'attenzione dell'esperto anche problemi più originali, ma il suo atteggiamento di fondo è sempre quello di cercare risposte che rimandino a regole rigide, nette, applicabili in modo abbastanza automatico. Spesso se non ottiene risposte di questo genere mette in discussione le risposte della redazione.

Il linguista ingenuo sembra molto sensibile soprattutto alla lingua scritta, e al tema della correttezza dei costrutti in essa usati. Per descrivere i fatti di lingua che sottopone al giudizio degli esperti usa una terminologia perlopiù tradizionale, anche se molto ricca. Un aspetto molto peculiare di questa rubrica è che gli utenti non solo cercano conferme sulla correttezza di un dato costrutto o di un uso linguistico, ma vogliono anche conferme sulla correttezza di analisi metalinguistiche (le tradizionali analisi grammaticale, logica e del periodo) che però talvolta risultano inutilmente complesse e non prive di inesattezze e insensatezze, retaggio della tradizione scolastica meno aggiornata.

Gli aspetti più interessanti emersi dall'analisi riguardano la sensibilità alla nozione di contesto d'uso, che però resta limitata al momento in cui si fornisce un esempio e non si estende a una considerazione più ampia per cui il riferimento al contesto d'uso non fa emergere l'idea che possano esistere regole variabili. Infine si è osservata qua e là una notevole sensibilità a fatti di lingua per la cui descrizione e definizione, però, il lettore medio non dispone di strumenti linguistici specialistici. 


\section{Bibliografia}

Achard-Bayle, Guy et Mariee- Anne Paveau (2008), «Présentation. La linguistique « hors du temple » », Pratiques, vol. 139/140, p. 3-16.

Albano Leoni, Federico (2015), «Carmniell o’ srngar. Osservazioni sulla ortografia ingenua del napoletano e sulle sue possibili implicazioni fonetiche» in Silvia Dal Negro, Federica Guerini e Gabriele Iannàccaro (ed.), Elaborazione ortografica delle varietà non standard. Esperienze spontanee in Italia e all'estero, Bergamo, Bergamo University Press, p. 51-78.

Antonelli, Giuseppe (2007), L'italiano nella società della comunicazione, Bologna, Il Mulino.

Antos, Gerd (1996), Laien-Linguistik: Studien zu Sprach- und Kommunikationsproblemen im Alltag. Am Beispiel von Sprachratgebern und Kommunikationstrainings, Tübingen, Niemeyer.

Biffi, Marco e Raffaella Setti (2013), La Crusca risponde. Dalla carta al web (1995-2005), Firenze, Le Lettere.

Blommaert, Jan (ed.) (1999), Language Ideological Debates, Germany, De Gruyter Mouton.

Blommaert, Jan (2006), «Language ideology», in Encyclopedia of Language \& Linguistics, Amsterdam, Elsevier, p. 510-522.

D’Achille, Paolo (2014), «Dove va l'italiano? Linee di tendenza della lingua di oggi», in Sergio Lubello (ed.), Lezioni di italiano. Riflessioni sulla lingua nel nuovo millennio, Bologna, Il Mulino, p. 13-36.

Demel, Daniela (2006), «Laienlinguistik und Sprachchroniken: Italienisch», in Gerhard Ernst, Martin-Dietrich Glessgen e Christian Schmitt (ed.), Romanische Sprachgeschichte: ein internationales Handbuch zur Geschichte der romanischen Sprachen, vol. 2, Berlin/New York, De Gruyter, p. 1523-1533.

Demel, Daniela (2007), Si dice o non si dice? Sprachnormen und normativer Diskurs in der italienischen Presse, Frankfurt, Peter Lang.

Dixon, Robert M. W. (1992), «Naive Linguistic Explanation», Language in Society, vol. 21/1, p. 83-91.

Doury, Marianne (2008), « «Ce n’est pas un argument! » Sur quelques aspects des théorisations spontanées de l'argumentation», Pratiques, vol. 139/140, p. 111-128.

Gal, Susan (2002), «Language Ideologies and Linguistic Diversity: Where Culture Meets Power», in László Kereszes e Sándor Maticsák (ed.), A Magyar Nyelv Idegenben, Debrecen, Debreceni Egyetem Finnugor Nyelvtudományi Tanszéke, p. 197-204.

Graffi, Giorgio (1991), «Concetti «ingenui» e concetti «teorici» in sintassi», Lingua e stile, vol. 26/1, p. 347-363.

Graffi, Giorgio (1994), Sintassi, Bologna, Il Mulino. 
Heath, Shirley Brice (1989), «Language ideology», in Erik Barnouw (ed.), International Encyclopedia of Communications, vol. 2, New York, Oxford University Press, p. 393-395.

Hoenigswald, Henry M. (1966), «A proposal for the study of folk linguistics», in William Bright (ed.), Sociolinguistics. Proceedings of the UCLA Sociolinguistics Conference 1964, The Hague-Paris, Mouton, p. 16-26.

Houdebine, Anne-Marie (1985), «Pour une linguistique synchronique dynamique», La linguistique, vol. 21, p. 7-36.

Houdebine, Anne-Marie (2002), «L'Imaginaire Linguistique et son analyse», Travaux de linguistique, vol. 7, p. 11-27.

Jaeckel, Volker e Rolf Kailuweit (2006), «Laienlinguistik und Sprachchroniken: Iberische Halbinsel und Lateinamerika», in Gerhard Ernst, Martin-Dietrich Glessgen, e Christian Schmitt (ed.), Romanische Sprachgeschichte: ein internationales Handbuch zur Geschichte der romanischen Sprachen, vol. 2, Berlin/New York, De Gruyter, p. 1546-1557.

Leone, Massimo (2011), «Dall'ideologia linguistica all'ideologia semiotica. Riflessioni sulla smentita», Esercizi Filosofici, vol. 6, p. 318-328.

Osthus, Dietmar (2006), «Laienlinguistik und Sprachchroniken: Französisch und Okzitanisch», in Gerhard Ernst, Martin-Dietrich Glessgen e Christian Schmitt (ed.), Romanische Sprachgeschichte: ein internationales Handbuch zur Geschichte der romanischen Sprachen, vol. 2, Berlin/New York, De Gruyter, p. 1533-1546.

Paveau, Marie-Anne (2008), «Les non-linguistes font-ils de la linguistique? Une approche anti-éliminativiste des théories folk.» Pratiques, vol. 139/140, p. 93-109.

Preston, Dennis R. (2008), «Qu'est-ce que la linguistique populaire ? Une question d'importance», Pratiques, vol. 139/140, p. 1-24.

Remysen, Wim (2011), «L'application du modèle de l'imaginaire linguistique à des corpus écrits: le cas des chroniques de langage dans la presse québécoise», Langage et Société, vol. 135, p. 47-65.

Remysen, Wim e Sabine Schwarze (2015), «Éditorial - présentation de la revue Circula», Circula, vol. 1, p. 1-3.

Sapir, Edward (1921), Language. An Introduction to the Study of Speech, New York, Harcourt, Brace and World.

Sapir, Edward (1933), «La réalité psychologique des phonèmes», Journal de Psychologie Normale et Pathologique, vol. 30, p. 247-265.

Schmale, Günter (2008), «Conceptions populaires de la conversation», Pratiques, vol. 139/140, p. 5880.

Serianni, Luca (2006), Prima lezione di grammatica, Bari, Laterza.

Serianni, Luca (2007), «La norma sommersa», Lingua e stile, vol. 42, p. 283-298. 
Sériot, Patrick, Elena Bulgakova e Andreja Eržen (2008), «La linguistique populaire et les pseudosavants», Pratiques, vol. 139/140, p. 149-162.

Setti, Raffaella (2011), «La consulenza linguistica», in Vittorio Coletti (ed.), L'italiano dalla nazione allo Stato. Firenze, Le Lettere, p. 261-274.

Stegu, Martin (2008), «Linguistique populaire, language awareness, linguistique appliquée: interrelations et transitions», Pratiques, vol. 139/140, p. 81-92.

Tassone, Dalila (2015), «La percezione della norma linguistica attraverso il periodico La Crusca per voi», Circula, vol. 1, p. 130-152.

Woolard, Kathryn A. (1992), «Language ideology. Issues and approaches», Pragmatics, vol. 2-3, p. 235 249.

Woolard, Kathryn A. (1998), «Introduction. Language ideology as a field of inquiry», in Bambi B. Schieffelin, Kathryn A. Woolard e Paul V. Kroskrity (ed.), Language ideologies. Practice and theory, New York/Oxford, Oxford University Press, p. 3-49. 